\title{
Percepción del Clima Escolar en Estudiantes de Enseñanza Media de Valparaíso de Colegios Municipales, Particulares Subvencionados y Particulares
}

\author{
School climate perceived in secondary students who study in public, subsidized and
} private schools in Valparaiso city

Percepção de ambiente social do centro escolar em estudantes do ensino medio da cidade de Valparaiso (Chile) de escolas municipais, subvencionadas e particulares

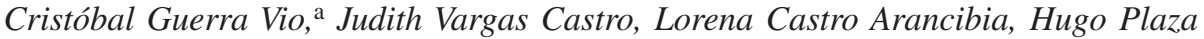 Villarroel y Paulina Barrera Montes}

aUniversidad Santo Tomás. Telf.: 09-95328046. Correo electrónico: cguerravio@yahoo.es

\begin{abstract}
RESUMEN
Se realizó una investigación en estudiantes de enseñanza media de la ciudad de Valparaíso (Chile), en que 1075 estudiantes de ambos sexos respondieron un cuestionario sobre clima social del centro escolar (CECSCE). Los estudiantes perciben en términos generales un clima social escolar moderado, ponderando mejor el clima referido al centro escolar con respecto al clima del profesorado. Se observaron diferencias en la opinión según el género del estudiante, donde las mujeres perciben el clima escolar de manera más favorable que sus pares varones. En cuanto a diferencias según tipo de establecimiento, los estudiantes de colegios municipalizados ponderaron de modo más desfavorable el clima social escolar que sus pares de colegios subvencionados y particulares. No se observaron efectos interactivos entre el género del estudiante y el tipo de establecimiento sobre la percepción de clima social escolar.
\end{abstract}

Palabras clave: clima escolar, estudiantes, enseñanza media, colegios de Valparaíso.

\begin{abstract}
An investigation was conducted in secondary school students who study in Valparaíso city. 1075 students (males and females) answered the School Environment Questionnaire (CECSCE). In general terms, students perceive a moderate social environment in the school. Additionally they considered than the school center atmosphere is better than the one of the school teachers. Furthermore, female students perceived better school climate than male students. Differences per type of school were analyzed. A worst social climate perception was observed in public schools in comparison with subsidized and private schools. There were not interactive effects between gender and type of school on perceptions of school social climate.
\end{abstract}

Key words: school social climate, students, secondary education, valparaíso schools.

\section{RESUMO}

Uma pesquisa foi realizada com estudantes do ensino médio da cidade de Valparaíso (Chile), em que 1075 estudantes responderam a um questionário sobre o ambiente social do centro escolar (CECSCE). Os estudantes percebem em geral um ambiente escolar médio, , sendo melhor o ambiente em relação ao centro escolar que o relacionado com os professores. Foram observadas diferenças na opinião de acordo com o gênero, sendo as mulheres as que percebem um ambiente escolar de maneira mais favorável se comparado com os homens. Em relação às diferenças por tipo de escolas, os estudantes de escolas municipais mostram uma percepção mais desfavorável do ambiente escolar que seus iguais de escolas subvencionadas e particulares. Além disto, não foram observados efeitos interativos entre o gênero do estudante e o tipo de escola em relação à percepção do ambiente social escolar.

Palavras chave: ambiência escolar, estudantes, ensino médio, colégios de valparaíso. 


\section{INTRODUCCIÓN}

En el contexto de la reforma del sistema educativo chileno, se ha venido hablando, desde hace ya varios años, de la necesidad de orientar las políticas educativas no sólo en lo académico, sino que considerando la necesidad de generar un adecuado clima de convivencia escolar que favorezca el proceso de enseñanza-aprendizaje y que potencie el desarrollo integral de los estudiantes (MINEDUC, 2002). En este sentido, surge con fuerza el concepto "clima escolar" como eje articulador de las distintas políticas educacionales.

El clima escolar corresponde a la percepción que los individuos tienen de los distintos aspectos del ambiente escolar (Arón y Milicic, 1999a). Infante et al. (2003) agregan que se refiere a la percepción favorable que alumnos y profesores tienen de la clase y de los lazos mutuos de apoyo, aceptación, reconocimiento y afecto. Arón y Milicic (1999b) proponen dos nomenclaturas para referirse al clima escolar, se refieren a un clima escolar tóxico cuando los estudiantes perciben al colegio como un lugar rígido, injusto y centrado en las descalificaciones entre sus agentes. En cambio, hablan de un clima escolar nutritivo cuando los estudiantes perciben justicia, reconocimiento y apoyo en el establecimiento educativo.

Distintos autores indican que los elementos personales, estructurales y funcionales del sistema educativo influyen en dicha percepción (CERE, 1993; Rodríguez, 2004). En este sentido, Cornejo y Redondo (2001) señalan que lo central del Clima Social de una institución es la percepción que tienen los sujetos acerca de las relaciones interpersonales que establecen en el contexto escolar (a nivel de aula o de centro educativo) y el contexto o marco en el cual estas instituciones se desenvuelven. De ahí que, para evaluar el clima escolar, sea necesario consultar a los sujetos que interactúan en él. Álvarez, Álvarez-García, González-Castro, Núñez y González-Pienda (2006) sugieren consultar directamente a los alumnos ya que éstos pueden informar tanto de las pautas de relación entre los estudiantes, muchas de ellas invisibles para padres y profesores, y de las relaciones profesor-alumno.

El interés por estudiar y favorecer un adecuado clima escolar radica en que ha sido asociado a una serie de beneficios para la comunidad educativa. Por ejemplo, un adecuado clima escolar (o clima escolar nutritivo) favorece la convivencia pacífica (Ma, 2008), motiva actividades educativas (Alcalay, Milicic, Torreti, 2005; Anderson et al., 2004) у modera sus problemas psicológicos (Westling, 2002). Por el contrario, cuando el clima escolar no es adecuado (clima tóxico) se asocia a mayores niveles de estrés, irritación, depresión, falta de motivación por parte de los estudiantes (Arón y Milicic, 1999a; Ascorra, Arias y Graff, 2003) y a altos índices de violencia escolar (Fernández et al., en prensa; Guerra, Castro y Vargas, 2011; Universidad Alberto Hurtado y Gobierno de Chile, 2006).

Los primeros reportes formales de estudios de clima escolar en Chile, publicados en revistas especializadas, se encuentran en el trabajo de Cornejo y Redondo (2001), quienes encuestaron a estudiantes de Enseñanza Media de la Región Metropolitana (de colegios municipales y particulares subvencionados) y describieron su percepción de clima escolar. Los estudiantes encuestados percibieron los más bajos niveles de clima escolar en el ámbito interpersonal (referido a la percepción de cercanía y preocupación por parte de los profesores) y en los ítems referidos a la percepción del colegio como una instancia estimulante (generadora de espacios de creatividad) y justa (en la aplicación de normas y sanciones). Por otro lado, los estudiantes percibieron un adecuado clima escolar referido al contexto instruccional (vinculado al favorecimiento del aprendizaje). Por esta razón, los 
autores concluyen que el sistema escolar estaría centrado en el favorecimiento de logros académicos más que en el logro de relaciones interpersonales satisfactorias. Por su parte, Vergara (s/f) realiza un estudio similar, pero en colegios municipales de la Región de la Araucanía. El autor concluye que la mayoría de los estudiantes encuestados perciben un clima social escolar favorable en lo referido a las relaciones interpersonales con los profesores y a la generación de un ambiente propicio para el proceso de enseñanzaaprendizaje. No obstante, señalan que perciben el contexto disciplinario como desfavorable.

Otro estudio fue desarrollado por IDEA (2005) en colegios municipales, particulares subvencionados y particulares de todo el país. El estudio concluye una convivencia escolar positiva (el $81 \%$ de los alumnos afirma sentirse muy bien y tener muchos amigos). Además, la mayoría de los alumnos señala que el establecimiento les enseña a relacionarse en forma positiva con los demás, la mayoría valora las relaciones que mantienen con sus compañeros y el orden que existe en sus establecimientos. Por otro lado, este estudio destaca que los aspectos del clima escolar peor valorados por los estudiantes están referidos a la percepción de los alumnos de ser poco considerados a la hora de resolver los problemas que se plantean en el colegio, de que existe disparidad de criterios por parte de los profesores en la aplicación de las normas (favoritismos). Además, este estudio concluye que los estudiantes de colegios municipales y particulares subvencionados perciben un clima escolar más adecuado que en el caso de los colegios particulares.

Por su parte, el informe Convivencia en el ámbito escolar (UNICEF y Time Research S.A., 2005), consideró a una muestra de estudiantes de $3^{\circ}$ y $4^{\circ}$ medio de Iquique, Santiago y Temuco (de establecimientos municipales, particulares subvencionados y particulares). Se concluye que, en general, los alumnos se sienten apoyados por sus profesores (sobre todo en los colegios particulares subvencionados) y por sus compañeros (sobre todo en los particulares). No obstante, se arrojan indicadores de un clima escolar inadecuado en lo referido a la percepción de discriminación (uno de cada tres niños señala haber sufrido algún tipo de discriminación en el colegio) y nivel de identificación con el colegio (el $62 \%$ de los estudiantes siente indiferencia hacia su colegio).

Murillo y Becerra (2009), mediante un estudio de redes semánticas, realizado en colegios municipales, particulares subvencionados y particulares de la ciudad de Temuco, concluyen que los estudiantes vinculan el clima escolar exclusivamente con los contextos de aprendizaje y no con el ámbito interpersonal. También se señala que los problemas de comunicación, los estilos de liderazgo inadecuados, la intolerancia, la envidia, el egoísmo y la irresponsabilidad son los obstaculizadores de un adecuado clima escolar.

Otros estudios chilenos han abordado indirectamente el estudio del clima escolar ligándolo al fenómeno de la violencia escolar. Es así como entre un $28 \%$ y un $47 \%$ de los estudiantes chilenos declara haber sido víctima directa de violencia escolar por parte de sus pares y entre un $46 \%$ y un $86 \%$ declara haber sido testigos de ella (Fleming y Jacobsen, 2006; Idea, 2005; UNICEF, 2004; Zerón, 2002). Además, un 11\% reporta haber sido agredido por un profesor (Universidad Alberto Hurtado y Gobierno de Chile, 2006). La mayoría de las investigaciones señala que la violencia escolar es más frecuente en establecimientos de nivel socioeconómico bajo (Fleming y Jacobsen, 2006; Guerra, Álvarez-García, Dobarro, Núñez, Castro y Vargas, 2010; Instituto Idea, 2005; Universidad Alberto Hurtado y Gobierno de Chile, 2006). Por otro lado, las agresiones entre pares más frecuentemente reportadas corresponden a agresiones psicológicas como gritos, burlas y descalificaciones (43\%), luego la agresión física (30\%) y la discriminación (12\%) 
(Universidad Alberto Hurtado y Gobierno de Chile, 2006). En relación a la violencia escolar ejercida por profesores en contra de alumnos, se observa que un $16 \%$ dice haber sido amenazado, un $23 \%$ insultado, un $40 \%$ ridiculizado y un $43 \%$ siente que los docentes le tienen "mala" (IDEA, 2005).

Es por esta razón que el presente estudio pretende describir la percepción del clima escolar de un grupo de estudiantes de enseñanza media de la ciudad de Valparaíso. Adicionalmente, se pretende comparar la percepción de los estudiantes por género y según tipo de establecimiento educacional (colegios municipales, particulares subvencionados y particulares). Sobre la base de los reportes de los estudios chilenos previos, se espera obtener resultados similares en la muestra de estudiantes de Valparaíso. Específicamente, se espera encontrar niveles generales adecuados de clima escolar. Respecto de diferencias por género, no se establecen pronósticos a priori, ya que no se cuentan con evidencias suficientes. Sobre las diferencias por tipo de establecimiento, se espera obtener una menor percepción de un clima escolar adecuado en estudiantes de colegios municipales, respecto de los colegios subvencionados y particulares.

Se considera relevante la realización de este tipo de investigaciones ya que, en Chile, existe escasez de estudios publicados en el tema, adicionalmente, se utiliza un instrumento de medición del clima escolar adaptado a la realidad chilena y con adecuados niveles de fiabilidad y validez.

\section{MÉTODO}

\subsection{PARTICIPANTES}

Mediante un muestreo intencionado, participaron 1075 estudiantes de Enseñanza Media de Valparaíso (45,5\% niñas y 54,5\% niños). La media de edad de los participantes fue de 15,98 años (D.T.=1,30). Pertenecían a 6 colegios con distinto tipo de dependencia (2 municipalizados, 2 particulares subvencionados y 2 particulares). El $43 \%$ de los participantes estudiaba en colegios municipalizados, el 29,9\% en colegios particulares subvencionados y el $27,2 \%$ en colegios particulares.

\subsection{INSTRUMENTOS}

a) Ficha de antecedentes personales: cada estudiante respondió una ficha de antecedentes de forma auto aplicada, donde debían señalar género, curso, edad, y tipo de establecimiento al cual pertenecían.

b) Cuestionario de Clima Social del Centro Escolar (CECSCE): este instrumento fue desarrollado y validado originalmente en España por Trianes, Blanca, de la Morena, Infante y Raya (2006). Posteriormente Guerra, Castro y Vargas (2010) lo adaptan a Chile, analizan sus propiedades psicométricas y concluyen que presenta adecuados índices de fiabilidad y validez (factorial y convergente).

El CECSCE es un instrumento de autorregistro que, en catorce ítems, evalúa dos dimensiones del clima escolar: El Clima escolar del Centro (referido a la capacidad de ayuda, seguridad, comodidad y respeto percibidos en el centro educativo) y el Clima escolar referente al Profesorado (referido a la percepción de los alumnos sobre el trato dado por los profesores). El formato de respuestas ofrece alternativas del 1 al 5. Para obtener el puntaje de cada dimensión se suman las respuestas de sus respectivos ítems. El 
puntaje de Clima del Centro oscila entre 8 y 40, mientras que en Clima del Profesorado lo hace entre 6 y 30. En ambos casos, se interpreta que a mayor puntaje, mayor es la percepción de un adecuado clima social escolar.

En este estudio se obtuvo una adecuada fiabilidad, obteniéndose un alfa de 0,83 para Clima del Centro y de 0,72 para Clima del Profesorado. Por su parte, el CECSCE total obtuvo un alfa de 0,86 .

\subsection{PROCEDIMIENTO}

Para valorar su viabilidad práctica y ética, el proyecto de investigación fue evaluado y aprobado por dos académicos de una de las universidades que patrocinaron el estudio, pero externos al equipo investigador. Luego, el equipo directivo de cada colegio fue informado de los objetivos y procedimientos del estudio, del carácter voluntario y anónimo de la participación por parte de los estudiantes y del tratamiento confidencial de los resultados. Los equipos directivos de todos los establecimientos consultados aceptaron participar del estudio. Los mismos colegios, asesorados por el equipo de investigación, gestionaron el consentimiento de los padres de los estudiantes que formaron parte del estudio. Los padres firmaron el consentimiento enviado mediante comunicación escolar, además, los alumnos fueron informados de los objetivos del estudio y del carácter voluntario, anónimo y confidencial de sus respuestas. Luego, se aplicó el instrumento a los estudiantes que, además de tener el consentimiento de sus padres, dieron su consentimiento verbal para formar parte del estudio. La aplicación fue colectiva, dentro del establecimiento y en horario de clases.

Una vez recogidos los datos, fueron analizados con el programa SPSS. Primero se examinó la distribución de los datos mediante la prueba de Kolmogorov-Smirnov y, dado que la mayoría de las variables no se ajustaron a una distribución normal, se trabajó con estadística no paramétrica. Luego se calculó el puntaje medio obtenido, en cada ítem y en cada dimensión del CECSCE, por la muestra total y por la muestra dividida según el tipo de colegio de procedencia; adicionalmente se calculó si existían diferencias significativas entre ambas escalas con la prueba de rangos de Wilcoxon. Seguidamente, se realizaron comparaciones por género con la prueba U de Mann-Withney y tipo de colegio con la prueba de Kruskal-Wallis. Para evaluar entre qué tipo específico de colegio se producían diferencias, se realizaron análisis con la prueba U de Mann-Withney, ajustando el nivel de significación según el criterio de Bonferroni. Finalmente, para evaluar si existían interacciones entre género y tipo de establecimiento sobre el clima escolar se optó por un análisis de varianza factorial $(3 \times 2)$.

\section{RESULTADOS}

\subsection{PERCEPCIÓN DEL CLIMA ESCOLAR DE ESTUDIANTES DE ENSEÑANZA MEDIA DE VALPARAÍSO}

En la Tabla 1 pueden observarse los puntajes medios obtenidos por la muestra total en el CECSCE y en sus subescalas. Comparando el puntaje medio obtenido por los estudiantes con el puntaje máximo que ofrece la escala, se puede apreciar que estos perciben un clima escolar moderado. Esta tendencia se mantiene tanto en lo referido al Clima del Centro, al Clima del Profesorado, como en la escala total. 


\begin{tabular}{|l|l|}
\hline & $\begin{array}{l}\text { Muestra total } \\
(\mathbf{n = 1 0 7 5 )}\end{array}$ \\
\hline Clima del Centro & $27,52(5,79)$ \\
\hline Clima del Profesorado & $20,08(4,29)$ \\
\hline Clima Escolar Total & $47,61(8,97)$ \\
\hline $\begin{array}{l}\text { Valores posibles entre 8 y 40 para CESCE Centro, entre 6 y 30 } \\
\text { para CESCE Profesorado y entre 14 y 70 para CESCE Total. A } \\
\text { mayor puntaje, mayor percepción de un adecuado clima escolar }\end{array}$ \\
\hline
\end{tabular}

Tabla 1. Media (y DE) de los estudiantes de Enseñanza Media de Valparaíso en las dimensiones CECSCE

En la Tabla 2 se ofrece el valor medio obtenido por los estudiantes de la muestra total en cada reactivo del CECSCE. Se aprecia que, en promedio, perciben "casi siempre" siete manifestaciones de un adecuado clima escolar (reflejadas en los ítems 1, 3, 4, 5, 8, 9 y 12), "algunas veces" seis manifestaciones de un adecuado clima escolar (reflejadas en los ítems 2, 6, 7, 10,11 y 13) y "casi nunca" una manifestación de adecuado clima escolar (ítem 14).

\begin{tabular}{|l|c|}
\hline & $\begin{array}{c}\text { Muestra total } \\
\text { (n=1075) }\end{array}$ \\
\hline Factor 1: Clima del Centro & $3,80(1,10)$ \\
\hline 1. Cuando hay una emergencia, hay alguien para ayudarme & $3,57(1,09)$ \\
\hline 5. El colegio está muy ordenado y limpio & $3,16(1,09)$ \\
\hline 6. Se puede confiar en la mayoría de la gente de este colegio & $3,23(0,85)$ \\
\hline 7. Los estudiantes realmente quieren aprender & $3,62(1,13)$ \\
\hline 9. Los estudiantes de todas las razas y clases sociales son respetados & $3,44(1,10)$ \\
\hline 10. Mi clase tiene un aspecto muy agradable & $3,09(1,08)$ \\
\hline 11. La gente de este colegio se cuida uno al otro & $3,62(1,11)$ \\
\hline 12. Mi colegio es un lugar muy seguro & $3,44(0,95)$ \\
\hline Factor 2: Clima del Profesorado & $3,65(1,04)$ \\
\hline 2. Los profesores de este colegio son agradables con los estudiantes & $3,50(1,06)$ \\
\hline 3. Trabajo en las tareas escolares & $3,62(1,14)$ \\
\hline 4. Cuando los estudiantes rompen las reglas son tratados justamente & $3,44(1,07)$ \\
\hline 8. Los profesores me dicen cuando hago un buen trabajo & $2,44(1,31)$ \\
\hline 13. Los profesores hacen un buen trabajo buscando a los alumnos problemáticos & \\
\hline 14. Me siento cómodo hablando con mis profesores de mis problemas & \\
\hline $\begin{array}{l}\text { Valores de la escala: 1= Nunca; 2= Casi nunca, pocas veces; 3= Algunas veces; 4= Casi siempre, muchas } \\
\text { veces; 5= Siempre. }\end{array}$ & \\
\hline
\end{tabular}

Tabla 2. Media (y DE) de los estudiantes de Valparaíso de Enseñanza Media en cada uno de los ítems del CECSCE 
Dado que se obtuvieron adecuados índices de fiabilidad (valores alfa) para cada escala del CECSCE, es que se procedió a obtener un puntaje ajustado de cada subescala, dividiendo el puntaje obtenido por el número de ítem que correspondía; de este modo, se obtuvieron dos puntajes equivalentes con la finalidad de comparar si dentro de la muestra existían diferencias significativas en la percepción del clima de centro y del profesorado.

Como se observa en la Tabla 3, al realizar esta comparación mediante la prueba de rangos de Wilcoxon $(\mathrm{Z}=-4.792)$, se aprecian diferencias estadísticamente significativas en la percepción de ambas dimensiones del clima escolar ( $\mathrm{p}=0.0001)$, donde el clima del centro es percibido de modo más favorable que el clima del profesorado.

\begin{tabular}{|l|l|l|l|}
\hline & $\begin{array}{l}\text { Clima Centro } \\
\text { Ponderado } \\
(\mathbf{n = 1 0 7 5})\end{array}$ & $\begin{array}{l}\text { Clima } \\
\text { Profesorado } \\
\text { Ponderado } \\
(\mathbf{n = 1 0 7 5})\end{array}$ & Wilcoxon \\
\hline $\begin{array}{l}\text { Media } \\
\text { DT }\end{array}$ & $\begin{array}{l}3,44 \\
(0.724)\end{array}$ & $\begin{array}{l}3,34 \\
(0.715)\end{array}$ & $\mathrm{Z}=-4.792^{*}$ \\
\hline $\begin{array}{l}\text { Diferencias estadísticamente significativas al nivel } \mathrm{p}<0,0001 \text { entre percepción clima centro y } \\
\text { percepción clima profesorado }\end{array}$ \\
\hline
\end{tabular}

Tabla 3. Comparación puntajes Clima de centro y Clima profesorado

\subsection{PERCEPCIÓN DEL CLIMA ESCOLAR DE ESTUDIANTES DE ENSEÑANZA MEDIA DE VALPARAÍSO DE SEGÚN GÉNERO}

Al comparar el puntaje obtenido por los estudiantes según género (Tabla 4), es posible señalar que existen diferencias estadísticamente significativas para la escala total y cada una de las subescalas. Clima del Centro, $\mathrm{U}=131328,5 ; \mathrm{p}<, 019$, Clima del Profesorado, $\mathrm{U}=125271,500 ; \mathrm{p}=, 0001$, y Clima Escolar Total, $\mathrm{U}=127028,000 ; \mathrm{p}<, 002$. Para cada una de las variables, las mujeres obtienen puntajes mayores que los hombres.

\begin{tabular}{|l|l|l|l|}
\hline & $\begin{array}{l}\text { Femenino. } \\
(\mathbf{n = 4 8 9})\end{array}$ & $\begin{array}{l}\text { Masculino. } \\
(\mathbf{n = 5 8 6})\end{array}$ & $\mathrm{U}$ \\
\hline Clima del Centro & 28 & 27,12 & $\mathrm{U}=131328,500^{*} ;$ \\
& $(5,62)$ & $(5,91)$ & \\
\hline Clima del Profesorado & 20,58 & 19,67 & $\mathrm{U}=125271,500^{*} ;$ \\
& $(4,13)$ & $(4,38)$ & \\
\hline Clima Escolar Total & 48,6 & 46,8 & $\mathrm{U}=127028,0^{*} ;$ \\
& $(8,71)$ & $(9,1)$ & \\
\hline
\end{tabular}

Tabla 4. Comparación por género de las subdimensiones del CECSCE y CECSCE total

\subsection{PERCEPCIÓN DEL CLIMA ESCOLAR DE ESTUDIANTES DE ENSEÑANZA MEDIA DE VALPARAÍSO DE DISTINTO TIPO DE COLEGIO}

Al comparar el puntaje medio obtenido por los estudiantes de distinto tipo de colegio con el puntaje máximo que ofrece la escala, se puede apreciar que éstos perciben un clima escolar moderado en el CESCE total y dividido por subescala. Esto, en general, se 
mantiene en los tres tipos de establecimiento educacional. La única excepción la constituye la baja puntuación media obtenida por los estudiantes de colegios municipales en Clima del Centro (ver Tabla 5).

Para realizar un análisis más riguroso que la simple comparación entre los puntajes obtenidos por la muestra con los puntajes máximos posibles de la escala, se realizaron análisis con la prueba de Kruskal-Wallis. Los resultados muestran que existen diferencias estadísticamente significativas en la percepción del clima escolar entre los tres grupos. Las diferencias son significativas para todas las variables: Clima del Centro, $\chi 2=145,397 ; \mathrm{p}$ $<, 001$; Clima del Profesorado, $\chi 2=24,205 ; \mathrm{p}<, 001$; Clima Escolar Total $\chi^{2}=103,224$; $\mathrm{p}<, 001$. Luego, para evaluar entre qué tipos de centro educativo específicamente existen estas diferencias, se realizaron análisis con la prueba U de Mann-Withney. Considerando que son tres tipos de colegio, para disminuir el riesgo del error Tipo I, se acompañó este análisis con la corrección de Bonferroni. De este modo, se consideraron significativas las diferencias con un nivel crítico menor a ,017 (obtenido de ,05/3) (ver Tabla 5).

Los resultados indican que los colegios municipalizados presentan diferencias estadísticamente significativas con los particulares subvencionados en Clima del Centro, $\mathrm{U}=51594,500 ; \mathrm{p}<, 017$, Clima del Profesorado, $\mathrm{U}=63124,500 ; \mathrm{p}<, 017$, y Clima Escolar Total, $\mathrm{U}=54464,500 ; \mathrm{p}<, 017$. En todas estas variables la percepción de un adecuado clima escolar es menor en los colegios municipalizados que en los particulares subvencionados. Además, los estudiantes de colegios municipales perciben niveles significativamente menores de un adecuado clima escolar (en todas sus dimensiones) que los de colegios particulares: Clima del Centro, $U=33359,500 ; p<, 017$, Clima del Profesorado, $\mathrm{U}=38805,500 ; \mathrm{p}<, 017$, Clima Escolar Total, $\mathrm{U}=54019,000 ; \mathrm{p}<, 017$. Por último, los estudiantes de colegios particulares subvencionados perciben niveles más bajos de un adecuado clima escolar que los de colegios particulares en dos de las tres variables medidas: Clima del Centro, $\mathrm{U}=37418,000 ; \mathrm{p}<, 017$ y Clima Escolar Total, $\mathrm{U}=39695,500 ; \mathrm{p}<, 017$. La excepción la constituye Clima del Profesorado, donde no se encontraron diferencias significativas entre colegios particulares subvencionados y particulares: Clima del Profesorado, $\mathrm{U}=44558,500$; ns.

\begin{tabular}{|l|l|l|l|l|}
\hline & $\begin{array}{l}\text { Mun. } \\
(\mathbf{n = 4 6 2})\end{array}$ & $\begin{array}{l}\text { Par. Sub. } \\
(\mathbf{n = 3 2 1})\end{array}$ & $\begin{array}{l}\text { Part. } \\
(\mathbf{n}=\mathbf{2 9 2})\end{array}$ & $\mathrm{U}$ \\
\hline Clima del Centro & 25,29 & 28,22 & 30,28 & $\mathrm{U}_{\mathrm{AB}}=51594,500^{*} ;$ \\
& $(5,78)$ & $(5,58)$ & $(4,54)$ & $\begin{array}{l}\mathrm{U}_{\mathrm{AC}}=33359,500^{*} ; \\
\mathrm{U}_{\mathrm{BC}}=37418,000^{*}\end{array}$ \\
\hline Clima del Profesorado & 19,35 & 20,45 & 20,84 & $\mathrm{U}_{\mathrm{AB}}=63124,500^{*} ;$ \\
& $(4,41)$ & $(4,18)$ & $(4,04)$ & $\begin{array}{l}\mathrm{U}_{\mathrm{AC}}=54294,000^{*} ; \\
\mathrm{U}_{\mathrm{BC}}=44558,500\end{array}$ \\
\hline Clima Escolar Total & 44,65 & 48,67 & 51,13 & $\mathrm{U}_{\mathrm{AB}}=54464,500^{*} ;$ \\
& $(9,05)$ & $(8,74)$ & $(7,45)$ & $\mathrm{U}_{\mathrm{AC}}=38805,500^{*} ;$ \\
& & & $\mathrm{U}_{\mathrm{BC}}=39695,500^{*}$ \\
\hline
\end{tabular}

Tabla 5. Media (y DE) de los estudiantes de Valparaíso (divididos por tipo de colegio) en las dimensiones CECSCE. Comparaciones U de Mann-Withney 
En la Tabla 6 se pueden ver los valores promedio de los estudiantes de cada tipo de colegio en cada uno de los reactivos del CECSCE. Los estudiantes de colegios municipales perciben "casi siempre" dos manifestaciones de un adecuado clima escolar (reflejadas en los ítems 3 y 8); "algunas veces" once manifestaciones de un adecuado clima escolar (reflejadas en los ítem 1, 2, 4, 5, 6, 7, 9, 10, 11, 12 y 13); y "casi nunca" una manifestación de adecuado clima escolar (ítem 14). Por su parte, los estudiantes de colegios particulares subvencionados perciben "casi siempre" ocho manifestaciones de un adecuado clima escolar (reflejadas en los ítems 1, 3, 4 5, 8, 9, 12 y 13); "algunas veces" cinco manifestaciones de un adecuado clima escolar (reflejadas en los ítems 2, 6, 7, 10 y 11); y "casi nunca" una manifestación de adecuado clima escolar (ítem 14). Finalmente, los estudiantes de colegios particulares perciben "casi siempre" diez manifestaciones de un adecuado clima escolar (reflejadas en los ítems 1, 2, 3, 4, 5, 8, 9, 10, 11 y 12); y "algunas veces" cuatro manifestaciones de un adecuado clima escolar (reflejadas en los ítems 6, 7, 13 y 14).

En la última columna de la Tabla 6 se entrega información referida a la comparación de la percepción de los estudiantes según su colegio de procedencia. Para lograr parsimonia sólo se muestran los valores U de Mann-Withney estadísticamente significativos. Como se aprecia, los estudiantes de colegios municipales perciben menores niveles de un adecuado clima escolar que los de colegios particulares subvencionados y de colegios particulares en la mayoría de los ítems del CECSCE. Específicamente, los estudiantes de colegios municipales perciben menores índices de un clima escolar adecuado que los de colegios particulares subvencionados en los ítems 1, 4, 5, 6, 8, 9, 11 y 12. Además, perciben menores índices de un clima escolar adecuado que los de colegios particulares en los ítem 1, 2, 4, 5, 6, 9, 10, 11, 12 y 14. Por su parte, los de colegios particulares subvencionados perciben menores índices de un adecuado clima escolar que los de colegios particulares en los ítems 2, 5, 7, 9, 10, 11, 12 y 14 .

\begin{tabular}{|c|c|c|c|c|}
\hline & $\begin{array}{c}\text { Mun. } \\
(\mathrm{n}=462)\end{array}$ & $\begin{array}{c}\text { Par. Sub. } \\
(\mathrm{n}=321)\end{array}$ & $\begin{array}{c}\text { Part. } \\
(\mathrm{n}=292)\end{array}$ & $\mathbf{U}$ \\
\hline \multicolumn{5}{|l|}{ Factor 1: Clima Referente del Centro } \\
\hline $\begin{array}{l}\text { 1. Cuando hay una emergencia, hay } \\
\text { alguien para ayudarme }\end{array}$ & $\begin{array}{c}3,45 \\
(1,15)\end{array}$ & $\begin{array}{c}3,96 \\
(1,04)\end{array}$ & $\begin{array}{c}4,15 \\
(0,90)\end{array}$ & $\begin{array}{l}\mathrm{U}_{\mathrm{AB}}=55549,500^{*} ; \\
\mathrm{U}_{\mathrm{AC}}=43967,500^{*}\end{array}$ \\
\hline $\begin{array}{l}\text { 5. El colegio está muy ordenado y } \\
\text { limpio }\end{array}$ & $\begin{array}{c}3,20 \\
(1,12)\end{array}$ & $\begin{array}{c}3,73 \\
(1,02)\end{array}$ & $\begin{array}{c}3,99 \\
(0,91)\end{array}$ & $\begin{array}{l}\mathrm{U}_{\mathrm{AB}}=54484,000^{*} ; \\
\mathrm{U}_{\mathrm{AC}}=40402,000^{*} ; \\
\mathrm{U}_{\mathrm{BC}}=40405,000^{*}\end{array}$ \\
\hline $\begin{array}{l}\text { 6. Se puede confiar en la mayoría de } \\
\text { la gente de este colegio }\end{array}$ & $\begin{array}{c}2,89 \\
(1,10) \\
\end{array}$ & $\begin{array}{c}3,31 \\
(1,09) \\
\end{array}$ & $\begin{array}{c}3,43 \\
(0,96) \\
\end{array}$ & $\begin{array}{l}\mathrm{U}_{\mathrm{AB}}=58938,500^{*} ; \\
\mathrm{U}_{\mathrm{AC}}=49418,500^{*} ;\end{array}$ \\
\hline $\begin{array}{l}\text { 7. Los estudiantes realmente quieren } \\
\text { aprender }\end{array}$ & $\begin{array}{c}3,22 \\
(0,93)\end{array}$ & $\begin{array}{c}3,15 \\
(0,81)\end{array}$ & $\begin{array}{c}3,33 \\
(0,74)\end{array}$ & $\mathrm{U}_{\mathrm{BC}}=41009,500^{*}$ \\
\hline $\begin{array}{l}\text { 9. Los estudiantes de todas las razas } \\
\text { y clases sociales son respetados }\end{array}$ & $\begin{array}{c}3,31 \\
(1,15)\end{array}$ & $\begin{array}{c}3,70 \\
(1,12)\end{array}$ & $\begin{array}{c}4,02 \\
(0,95)\end{array}$ & $\begin{array}{l}\mathrm{U}_{\mathrm{AB}}=60440,500^{*} ; \\
\mathrm{U}_{\mathrm{AC}}=44040,500^{*} ; \\
\mathrm{U}_{\mathrm{BC}}=39759,500^{*}\end{array}$ \\
\hline $\begin{array}{l}\text { 10. Mi clase tiene un aspecto muy } \\
\text { agradable }\end{array}$ & $\begin{array}{c}3,34 \\
(1,10)\end{array}$ & 3,36 & 3,67 & $\begin{array}{l}\mathrm{U}_{\mathrm{AC}}=56443,500^{*} ; \\
\mathrm{U}_{\mathrm{BC}}=40119,500 *\end{array}$ \\
\hline $\begin{array}{l}\text { 11. La gente de este colegio se cuida } \\
\text { uno al otro }\end{array}$ & $\begin{array}{c}2,76 \\
(1,11)\end{array}$ & $\begin{array}{c}3,19 \\
(1,00)\end{array}$ & $\begin{array}{c}3,50 \\
(0,96)\end{array}$ & $\begin{array}{l}\mathrm{U}_{\mathrm{AB}}=57869,500^{*} ; \\
\mathrm{U}_{\mathrm{AC}}=42507,000^{*} ; \\
\mathrm{U}_{\mathrm{BC}}=38885,500^{*}\end{array}$ \\
\hline
\end{tabular}




\begin{tabular}{|c|c|c|c|c|}
\hline $\begin{array}{l}\text { 12. Mi colegio es un lugar muy } \\
\text { seguro }\end{array}$ & $\begin{array}{c}3,12 \\
(1,08)\end{array}$ & $\begin{array}{c}3,82 \\
(1,04)\end{array}$ & $\begin{array}{c}4,21 \\
(0,84)\end{array}$ & $\begin{array}{l}\mathrm{U}_{\mathrm{AB}}=47262,000^{*} ; \\
\mathrm{U}_{\mathrm{AC}}=30458,000^{*} \\
\mathrm{U}_{\mathrm{BC}}=37385,000^{*}\end{array}$ \\
\hline \multicolumn{5}{|l|}{ Factor 2: Clima del Profesorado } \\
\hline $\begin{array}{l}\text { 2. Los profesores de este colegio son } \\
\text { agradables con los estudiantes }\end{array}$ & $\begin{array}{c}3,26 \\
(1,01) \\
\end{array}$ & $\begin{array}{c}3,45 \\
(0,90) \\
\end{array}$ & $\begin{array}{c}3,71 \\
(0,83) \\
\end{array}$ & $\begin{array}{l}\mathrm{U}_{\mathrm{AC}}=51157,000^{*} ; \\
\mathrm{U}_{\mathrm{BC}}=39334,500^{*}\end{array}$ \\
\hline 3. Trabajo en las tareas escolares & $\begin{array}{c}3,60 \\
(1,08) \\
\end{array}$ & $\begin{array}{c}3,71 \\
(1,04) \\
\end{array}$ & $\begin{array}{c}3,65 \\
(0,96) \\
\end{array}$ & \\
\hline $\begin{array}{l}\text { 4. Cuando los estudiantes rompen } \\
\text { las reglas son tratados justamente }\end{array}$ & 3,37 & 3,58 & 3,62 & $\begin{array}{l}\mathrm{U}_{\mathrm{AB}}=66900,500^{*} ; \\
\mathrm{U}_{\mathrm{AC}}=60007,500 *\end{array}$ \\
\hline $\begin{array}{l}\text { 8. Los profesores me dicen cuando } \\
\text { hago un buen trabajo }\end{array}$ & $\begin{array}{c}3,50 \\
(1,18) \\
\end{array}$ & $\begin{array}{c}3,77 \\
(1,07) \\
\end{array}$ & $\begin{array}{c}3,63 \\
(1,13) \\
\end{array}$ & $\mathrm{U}_{\mathrm{AB}}=64928,500^{*} ;$ \\
\hline $\begin{array}{l}\text { 13. Los profesores hacen un buen } \\
\text { trabajo buscando a los alumnos } \\
\text { problemáticos }\end{array}$ & $\begin{array}{c}3,39 \\
(1,09)\end{array}$ & $\begin{array}{c}3,53 \\
(1,09)\end{array}$ & $\begin{array}{c}3,44 \\
(1,00)\end{array}$ & \\
\hline $\begin{array}{l}\text { 14. Me siento cómodo hablando con } \\
\text { mis profesores de mis problemas }\end{array}$ & $\begin{array}{c}2,23 \\
(1,27)\end{array}$ & $\begin{array}{c}2,41 \\
(1,32)\end{array}$ & $\begin{array}{c}2,80 \\
(1,31)\end{array}$ & $\begin{array}{l}\mathrm{U}_{\mathrm{AC}}=50664,500 * \\
\mathrm{U}_{\mathrm{BC}}=38892,500^{*}\end{array}$ \\
\hline \multicolumn{5}{|c|}{$\begin{array}{l}\text { Valores de la escala: } 1=\text { Nunca; } 2=\text { Casi nunca, pocas veces; } 3=\text { Algunas veces; } 4=\text { Casi siempre, } \\
\text { muchas veces; } 5=\text { Siempre. } \\
\mathrm{AB}^{*}, \mathrm{AC}^{*} \text { y } \mathrm{BC}^{*} \text { : diferencias estadísticamente significativas al nivel } \mathrm{p}<0,017 \text { entre colegios tipo A } \\
\text { (Municipalizados), B (Particulares Subvencionados) y C (Particulares). }\end{array}$} \\
\hline
\end{tabular}

Tabla 6. Media (y DE) de los estudiantes de Valparaíso (divididos por tipo de colegio) en cada uno de los ítems del CECSCE. Comparaciones U de Mann-Withney

\subsection{EFECTO GÉNERO Y TIPO DE ESTABLECIMIENTO DEL ESTUDIANTE SOBRE LA PERCEPCIÓN DEL CLIMA ESCOLAR EN ESTUDIANTES DE ENSEÑANZA MEDIA DE VALPARAÍSO}

Para determinar si existían efectos de interacción entre el tipo de establecimiento y el género del alumno sobre la percepción de clima escolar, se procedió al cálculo de Anova Factorial para clima del centro, clima del profesorado y escala CECSCE total. Los resultados señalan que, para el caso del clima del centro, el modelo de dos factores (género $\mathrm{x}$ tipo de establecimiento) es estadísticamente significativo $(\mathrm{p}=0,0001)$ y logra explicar un 13,2\% de varianza; se observan los efectos principales ya detallados para género $(\mathrm{p}=0,0001)$ y tipo de establecimiento $(\mathrm{p}=0,006)$, no obstante, no hay efecto interactivo entre el género del estudiante y el tipo de establecimiento al cual pertenecen ( $\mathrm{F}=0,024$; $\mathrm{p}=0,976$ ), lo cual implica que las diferencias observadas en la percepción de hombres y mujeres es independiente al tipo de establecimiento al cual pertenecen.

Para el caso del clima del profesorado, el modelo de dos factores (género x tipo de establecimiento) es estadísticamente significativo ( $\mathrm{p}=0,0001)$ y logra explicar sólo un 3,1\% de varianza; se observan los efectos principales ya detallados para género $(\mathrm{p}=0,0001)$ y tipo de establecimiento ( $\mathrm{p}=0,0001)$, no obstante, no hay efecto interactivo entre el género del estudiante y el tipo de establecimiento al cual pertenecen $(\mathrm{F}=0,501 ; \mathrm{p}=0,606)$, lo 
cual implica que las diferencias observadas en la percepción de hombres y mujeres es independiente al tipo de establecimiento al cual pertenecen.

Finalmente, para la escala CESCE total, el modelo de dos factores (género x tipo de establecimiento) es estadísticamente significativo ( $\mathrm{p}=0,0001)$ y logra explicar sólo un $9,9 \%$ de varianza; se observan los efectos principales ya detallados para género $(\mathrm{p}=0,0001)$ y tipo de establecimiento $(\mathrm{p}=0,0001)$, no obstante, no hay efecto interactivo entre el género del estudiante y el tipo de establecimiento al cual pertenece $(\mathrm{F}=0,176$; $\mathrm{p}=0,838$ ), lo cual implica que las diferencias observadas en la percepción de hombres y mujeres es independiente al tipo de establecimiento al cual pertenecen.

\section{DISCUSIÓN}

El presente artículo tuvo como objetivos el describir la percepción del clima escolar de estudiantes de enseñanza media Valparaíso y comparar la percepción de estos estudiantes según género y el tipo de colegio al que asisten.

Los resultados de los análisis de datos con la muestra total indican que los estudiantes perciben un clima escolar moderado (tanto en lo referido al Clima del Centro, Clima del Profesorado y Clima Escolar Total). Estos resultados son acordes a lo esperado y coherentes con estudios previos que hablan de niveles favorables de clima escolar (Idea, 2005). Al analizar cada reactivo del CECSCE, en la muestra total, se aprecia que los ítems que reflejan la percepción de un adecuado clima social escolar están referidos, por un lado, a la seguridad, orden, limpieza y respeto que existe en el colegio, y por otro lado, a la percepción de los estudiantes de ser tratados justamente por sus profesores, de comprometerse en las tareas escolares y de ser reconocidos por ello. Los ítems que reflejan un moderado clima escolar se refieren al compromiso por aprender y al buen trato, confianza y cuidado mutuo existente entre los miembros de la comunidad educativa. Por último, se aprecia que los estudiantes casi nunca se sienten cómodos hablando con los profesores de sus problemas; lo anterior difiere en parte de los estudios previos que reportan un buen clima escolar en lo referido a la potenciación del aprendizaje y un peor clima escolar en lo referido a las relaciones interpersonales (Cornejo y Redondo, 2001; Murillo y Becerra, 2009; Vergara s/f). En la muestra se evidenció una mejor evaluación de clima de centro respecto del clima del profesorado, ante el cual los alumnos perciben no tener la confianza para plantear sus problemas; este hallazgo es coherente con lo planteado por Cornejo y Redondo (2001), y adicionalmente es relevante dada la necesaria interacción y confianza profesor-alumno para reportar hechos de violencia escolar y la resolución constructiva de conflictos (Fleming y Jacobsen, 2006; Idea, 2005; UNICEF, 2004; Zerón, 2002); adicionalmente, una baja percepción de confianza en el profesorado despersonaliza la acción educativa, centrando el foco en la tarea.

Al analizar las diferencias según género, se observa que los estudiantes varones perciben el clima escolar de manera más desfavorable que las mujeres. Este hallazgo tiene la relevancia de que, anteriormente, no se contaba con datos para poder plantear hipótesis sobre tales diferencias. Adicionalmente, se observó efecto de interacción entre el género y el tipo de establecimiento sobre las variables de estudio; al parecer la opinión por género es independiente del tipo de establecimiento; de cualquier manera, cabe señalar que el aporte de ambas variables en conjunto explica una pequeña proporción de 
varianza de las variables estudiadas, lo cual es coherente si se considera el clima escolar como un fenómeno multidimensional.

Por otro lado, al analizar las diferencias según el distinto tipo de colegio se observa que los estudiantes de colegios municipalizados presentan una percepción del clima escolar más desfavorable que los de colegios particulares subvencionados y particulares. Además se aprecia que los estudiantes de colegios particulares son los que perciben mayores índices de un adecuado clima escolar. Lo anterior concuerda con lo planteado en las hipótesis iniciales del estudio y confirman hallazgos previos (Fleming y Jacobsen, 2006; Guerra, Álvarez-García, Dobarro, Núñez, Castro y Vargas, 2010; Instituto Idea, 2005; Universidad Alberto Hurtado y Gobierno de Chile, 2006).

Este resultado es una evidencia más asociada a condiciones propias de los centros de dependencia municipalizada, ya que, de acuerdo a Aarón y Milicic (2010), en estos centros se aprecian condiciones materiales inadecuadas, estilos de liderazgo autoritarios, mayores niveles de violencia escolar, percepción desmejorada de capacitación de profesores y mayores niveles de burnout en profesores.

Si bien los resultados no arrojaron un clima escolar percibido inadecuado, tampoco se evidenció un clima escolar favorable en los establecimientos encuestados. Asimismo, las diferencias encontradas según tipo de colegio, ofrecen una evidencia más de aspectos desiguales en los tipos de colegio según dependencia administrativa, tales como migración de alumnos del sistema público al privado, incremento de rendimiento académico hacia el sector particular y mayor concentración de recursos.

\section{REFERENCIAS BIBLIOGRÁFICAS}

Alcalay, L., Milicic, N., Torretti, A. (2005). Alianza Efectiva Familia-Escuela: Un Programa Audiovisual Para Padres. Psykhe, 14, 2, 149-161.

Arón, A.M. y Milicic, N. (1999a). Clima social escolar y desarrollo personal. Un programa de mejoramiento. Santiago: Editorial Andrés Bello.

Arón, A.M. y Milicic, N. (1999 b). Climas sociales tóxicos y climas sociales nutritivos para el desarrollo personal en el contexto escolar. Psykhé, 2, 9, 117-123.

Arón, A. \& Milicic, N. (2000). Desgaste profesional de los profesores y clima social escolar. Santiago Chile: Ediciones Pontificia Universidad Católica de Chile.

Álvarez, L., Álvarez-García, D., González-Castro, P., Núñez, J.C., y González-Pienda, J. (2006). Evaluación de los comportamientos violentos en los centros educativos. Psicothema, 18, 686- 695.

Anderson, A.R., Christenson, S.L., Sinclair, M.F., y Lehr, C.A. (2004). Check and connect: The importance of relationships for promoting engagement with school. Journal of School Psychology, 42, 95-113.

Ascorra, P., Arias, H. y Graff, C. (2003). La escuela como contexto de contención social y afectiva. Revista Enfóques Educacionales 5, 1, 117-135.

CERE (1993). "Evaluar el contexto educativo", Documento de estudio. Vitoria: Ministerio de Educación y Cultura. Gobierno Vasco.

Cornejo, R. y Redondo, J. (2001). El clima escolar percibido por los alumnos de enseñanza media. Una investigación en algunos liceos de la Región Metropolitana. Última Década, 15, 11-52.

Fernández, F.J., Trianes, M.V., de la Morena, L., Escobar, M., Infante, L., y Blanca, M. J. (en prensa). Propiedades psicométricas de un cuestionario para la evaluación de la violencia cotidiana entre iguales en el contexto escolar. Anales de Psicología. 
Fleming, L.C., y Jacobsen, K.H. (2009). Bullying and symptoms of depression in Chilean middle school students. Journal of School Health, 79, 130-137.

Guerra, C., Álvarez-García, D., Dobarro, A., Núñez, J.C., Castro., L. y Vargas, J. (2011). Violencia escolar en estudiantes secundarios de Valparaíso (Chile): Comparación con una muestra española. Revista Iberoamericana de Psicología y Salud, 2, 1, 75-98.

Guerra, C., Castro, L. y Vargas, J. (2011). Examen psicométrico del Cuestionario de Clima Social del Centro Escolar en estudiantes chilenos. Psicothema, 23, 1, 140-145.

IDEA (2005). Primer estudio nacional de convivencia escolar. La Opinión de Estudiantes y Docentes 2005. Recuperado el 10 de mayo de 2010, de http://www.bligoo.com/media/users/1/91943/ files/primer\%20estudio\%20nacional\%20de\%20violencia\%20escolar\%20(informe_ejecutivo_estudio).pdf

Infante, L., Hierrezuelo, L., García, B., Sánchez, A., De la Morena, M.L., Muñoz, A., y Trianes, M.V. (2003). Evaluación de actitudes violentas y clima escolar en situaciones de agresividad en alumnado de Secundaria. Psicología, Saúde \& Doenças, 4, 2, 277-286.

Ma, L. (2008). The development of academic competence among adolescents who bully and who are bullied. Dissertation Abstracts International: Section B: The Sciences and Engineering, 68 (7-B), 4866.

MINEDUC (2002). Política de Convivencia Escolar. Recuperado el 25 de julio de 2006 de http:// www.mineduc.cl/biblio/documento/1197_Politica_Convivencia.pdf

Murillo, P. y Becerra, S. (2009). Las percepciones del clima escolar por directivos, docentes y alumnado mediante el empleo de "redes semánticas naturales". Su importancia en la gestión de los centros educativos. Revista de Educación, 350, 375-399

Rodríguez, N. (2004). El Clima Escolar. Revista Digital Investigación y Educación, 7, 3. Extraído el 9 de noviembre de 2010 desde http://www.csi-csif.es/andalucia/modules/mod_sevilla/archivos/ revistaense/n7v3/clima.PDF

UNICEF y Time Research S.A. (2005). Convivencia en el ámbito escolar. Informe cuantitativo primera parte. Recuperado el 10 de mayo de 2010, de http://www.unicef.cl/voz/docs/estudio_convivencia_escolar.pps

Universidad Alberto Hurtado y Gobierno de Chile (2006). Principales resultados del estudio nacional de Violencia en el Ámbito Escolar. Extraído del 13 de enero de 2010 desde http://www. educarchile.cl/UserFiles/P0001/File/CR_Articulos/Presentacion\%20Estudio\%20Nacional\%20 de\%20Violencia\%20Escolar_2006.pdf

Westling, M. (2002). A two level analysis of classroom climate in relation to social context, group composition and organization of special support. Learning Enviromments Research, 5, 253-274.

Vergara, R. (s/f). Clima social escolar en los centros educativos municipales de la Comuna de Toltén, Región de la Araucanía, Chile. Extractado el 5 de junio de 2011 desde http://www.liceus. com/cgi-bin/ac/pu/Clima\%20Social\%20Escolar,\%20La\%20Araucan\%C3\%ADa,\%20Chile.pdf 